\title{
Scaling up Health Informatics
}

\author{
Antoine Geissbuhler, President of IMIA \\ Geneva University Hospitals, Switzerland
}

It took decades for the work of enlightened pioneers in biomedical informatics to reach the mainstream in hospitals and outpatient care. Initial successful projects, those that were able to demonstrate impact on quality and efficiency of care, relied on locally-developed systems, painstakingly tuned to the socio-technical specificities of their organizations. It took two additional decades to reach the industrial stage where systems could be purchased and deployed without having to be reinvented each time.

The widespread - or looming - failure of healthcare systems has increased the need for quality and timely information in order to better navigate in these troubled systems. Healthcare information technologies being considered by many to be a remedy, their providers are under pressure to deliver solutions, and implementers expected to make them work in challenging environments.

Meanwhile, the democratization of the information society is bringing pervasive computing, connectivity and mobility, features and convenience that professionals and citizens are increasingly expecting to be able to use for healthcare and health management.

These combined forces tend to generate excitement and creativity, but, as the regulation of healthcare information technology is still rather immature, they also lead to a flood of tools and systems, mostly independent from each other. At a time where coordination of care, economies of scale, and strengthening of health systems are needed, this is deleterious.

In Uganda, for example [1], where dozens of eHealth and mHealth systems were being piloted or deployed without coordination, the government took the courageous decision to suspend the projects until they would be aligned to fit within the national eHealth strategy. This is, so far, a rather isolated ex- ample, but it illustrates the risks of "pilotitis" - the multiplication of pilot projects, many of which are unable to scale up and sustain. In wealthier settings, regulators also struggle to steer towards coherent systems and their meaningful use.

Indeed, key requirements are needed in order to deliver the promise of health informatics, as expressed in the IMIA vision:

"IMIA's vision is that there will be a world-wide systems approach for healthcare. Clinicians, researchers, patients and people in general will be supported by informatics tools, processes and behaviors that make it easy to do the right thing, in the right way, at the right time to improve health care for all. This systems approach will incorporate and integrate research, clinical care and public health." [2].

These include consistency, coherence, and interoperability of systems, access and responsible use of health data, capacity building of health informatics professionals, and the demonstration of impact through evaluation. These four domains have seen significant activity within IMIA this year.

The need for interoperability and standards is gaining renewed visibility, in particular through the preparation, with IMIA's support, of a new eHealth resolution [3] focusing on these aspects, which will hopefully be adopted by the World Health Assembly in May 2013. Preparatory meetings have demonstrated a significant momentum towards better collaboration and inclusion of developing countries, and highlighted the enabling role of IMIA.

In the context of the rapid development of big data analytics, IMIA's ongoing initiative on "transnational and trustworthy health data reuse" [4] aims at fostering a constructive dialogue between the industry, academia and the policy makers, in order to facilitate access to health data for public health and research, while guaranteeing the protection and the interests of citizen and other stakeholders. Capacity building for these issues is essential and should be integrated in multiple educational curricula, leveraging IMIA's leadership in education in health informatics and its accreditation of training programmes.

These efforts will only make sense if their impact can be measured and documented. Strong evidence of impact is still scarce, but convergence on standards and interoperable systems should lead to more consistent data and enable better studies. IMIA, jointly with WHO and ITU, is consolidating a tool box that will facilitate this convergence, thanks to expertise from several working group in IMIA and its regional organizations.

This long road to scalable, evidence-based health informatics will take us to Copenhagen for Medinfo 2013, where our global community will meet to work on "conducting medical informatics by converging technologies, conveying sciences and connecting people", a conference theme that very much embodies the essence and vision of IMIA.

\section{References}

1. Uganda mHealth moratorium, http:// www.ictworks.org/2012/02/22/ugandan-mhealth-moratorium-good-thing/, last accessed on April 15, 2013.

2. Murray PJ. The IMIA Strategic Plan - Towards IMIA 2015. IMIA Yearbook of Medical Informatics 2008. Methods Inf Med 2008;47 Suppl 1:7-15

3. eHealth standardization and interoperability, http://apps.who.int/gb/ebwha/pdf_files/ EB132/B132_R8-en.pdf, last accessed on April 15, 2013.

4. Geissbuhler A, Safran C, Buchan I, Bellazzi R, Labkoff S, Eilenberg K, et al. Trustworthy reuse of health data: a transnational perspective. Int $\mathrm{J}$ Med Inform 2013 Jan;82(1):1-9. 